\title{
NOTE ON STRICHARTZ INEQUALITIES FOR THE WAVE EQUATION WITH POTENTIAL
}

\author{
Seongyeon Kim, Ihyeok Seo And Jihyeon Seok
}

Abstract. We obtain Strichartz inequalities for the wave equation with potentials which behave like the inverse square potential $|x|^{-2}$ but might be not a radially symmetric function.

Mathematics subject classification (2010): 35B45, 35L05.

Keywords and phrases: Strichartz estimates, wave equation.

\section{REFERENCES}

[1] M. Beals And W. Strauss, $L^{p}$ estimates for the wave equation with a potential, Comm. Partial Differential Equations 18 (1993), 1365-1397.

[2] N. Burq, F. Planchon, J. G. Stalker And A. S. TahVildar-Zadeh, Strichartz estimates for the wave and Schrödinger equations with the inverse-square potential, J. Funct. Anal. 203 (2003), $519-549$.

[3] N. Burq, F. Planchon, J. G. Stalker and A. S. TahVildar-Zadeh, Strichartz estimates for the wave and Schrödinger equations with potentials of critical decay, Indiana Univ. Math. J. 53 (2004), $1665-1680$.

[4] J. Cheeger and M. TAYlor, On the diffraction of waves by conical singularities. I, Comm. Pure Appl. Math. 35 (1982), 275-331.

[5] M. Christ And A. Kiselev, Maximal functions associated to filtrations, J. Funct. Anal. 179 (2001), $409-425$.

[6] S. Cuccagna, On the wave equation with a potential, Comm. Partial Differential Equations 25 (2000), 1549-1565.

[7] V. Georgiev and N. Visciglia, Decay estimates for the wave equation with potential, Comm. Partial Differential Equations 28 (2003), 1325-1369.

[8] M. Goldberg, L. Vega And N. Visciglia, Counterexamples of Strichartz inequalities for Schrödinger equations with repulsive potentials, Int. Math. Res. Not. 2006, Art. ID 13927, 16pp.

[9] M. KeEl And T. TaO, Endpoint Strichartz estimates, Amer. J. Math. 120 (1998), 955-980.

[10] H. LindBLAD AND C. D. SOGGE, On existence and scattering with minimal regularity for semilinear wave equations, J. Funct. Anal. 130 (1995), 357-426.

[11] F. Planchon, J. G. Stalker and A. S. TAhVildar-ZAdeh, $L^{p}$ estimates for the wave equation with the inverse-square potential, Discrete Contin. Dyn. Syst. 9 (2003), 427-442.

[12] A. RUIZ AND L. VEGA, Local regularity of solutions to wave equation with time-dependent potentials, Duke Math. J. 76 (1994), 913-940.

[13] R. STRICHARTZ, Restrictions of Fourier transforms to quadratic surfaces and decay of solutions of wave equations, Duke Math. J. 44 (1977), 705-714.

[14] J. L. VAZQueZ AND E. ZuAZuA, The Hardy inequality and the asymptotic behaviour of the heat equation with an inverse-square potential, J. Funct. Anal. 173 (2000), 103-153. 